\title{
MIGRATIONS ET STRATÉGIES DES FAMILLES MOZAMBICAINES : RÉFLEXIONS POUR UNE POLITIQUE INTÉGRÉE DE DÉVELOPPEMENT RURAL
}

\author{
Sara Mercandalli", Ward Anseeuw ${ }^{* *}$
}

Dans le contexte démo-économique mozambicain et de libéralisation postapartheid, l'évolution des stratégies des familles rurales vers des systèmes d'activités composites et l'importance des migrations de travail soulignent les enjeux des transformations socioéconomiques des communautés rurales d'origine. À partir du cas de la localité de Leonzoane située dans la région sud, cet article montre que les migrations de travail des familles sont un élément central de recomposition de leurs systèmes d'activités et du marché du travail rural. Ces recompositions questionnent les fondements de la stratégie nationale de lutte contre la pauvreté et de développement rural (2011-2014) et appellent une approche intégrée prenant mieux en compte les dimensions des migrations et de l'emploi rural.

Mots-clés : Système familial d'activités, migration, marché du travail rural, politique de développement rural, Mozambique.

En Afrique subsaharienne, différentes études mettent en évidence la diversité du marché du travail rural (Sender et al., 2006 ; Oya, 2010), ainsi que l'importance des migrations de travail dans l'évolution des stratégies des familles (Black et al., 2006b). Elles soulignent ainsi leurs enjeux dans le cadre des transformations socioéconomiques à l'œuvre en zones rurales et alimentent le débat, à la fois

\footnotetext{
* Économiste du développement ; Cirad UMR ART-Dev., Montpellier. sara.mercandalli@cirad.fr.

** Économiste du développement ; Cirad UMR ART-Dev et université de Prétoria, Post-Graduate School of Agriculture and Rural Development, ward.anseeuw@up.ac.za.
} 
scientifique et politique, sur le rôle du travail salarié et des migrations de travail dans les dynamiques de la pauvreté et du développement rural.

Figure 1 : Situation de la localité de Leonzoane, Mozambique

Source : UN, 2004.

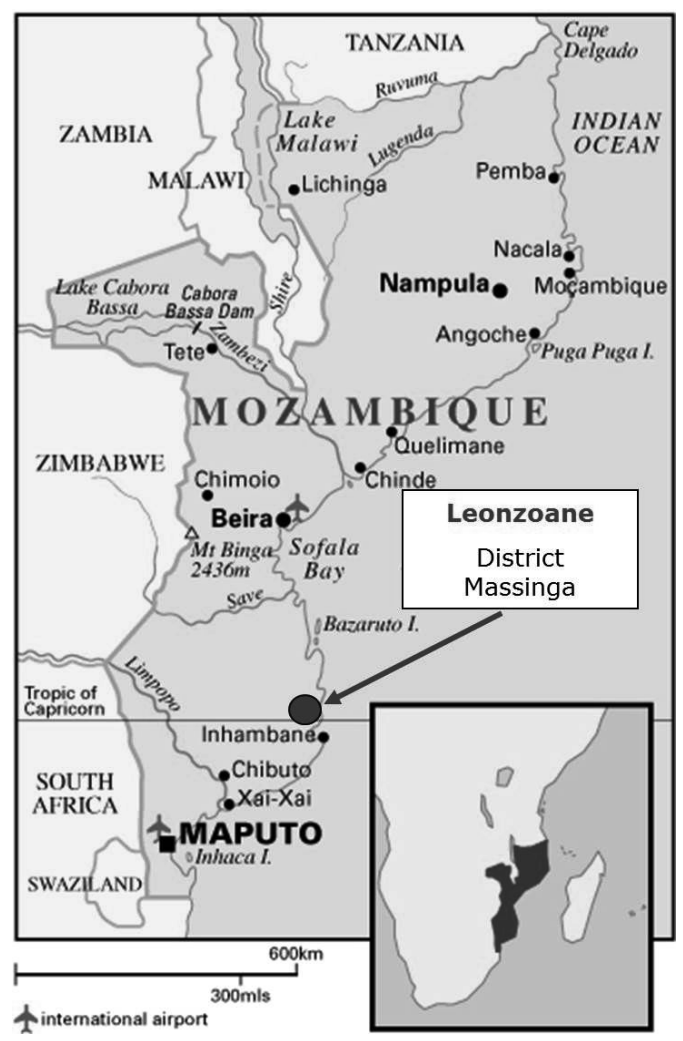

C'est particulièrement le cas dans le contexte démographique et économique du Mozambique qui, depuis la libéralisation postapartheid sud-africaine et régionale, connaît une évolution des stratégies des familles rurales vers des systèmes d'activités composites. Au-delà de la diversité des marchés du travail auxquels les familles participent (Massingarela et al., 2005), l'importance des migrations de travail au sein de ces stratégies (De Vletter, 2007) questionne leur rôle comme élément structurant des systèmes d'activités des familles et aussi comme élément de transformations socioéconomiques des communautés rurales d'origine. Ces transformations sont à resituer dans le contexte des changements successifs du cadre migratoire national avec le travail forcé dans les mines sud-africaines jusqu'à l'Indépendance (1975), la période de guerre civile qui a déplacé une partie importante de la population (1977-1992) puis la fin de l'apartheid (1994). Tenir compte de ces recompositions est donc primordial 
dans le cadre de la nouvelle stratégie nationale de lutte contre la pauvreté du pays (2011-2014).

Ainsi, à partir du cas de la localité de Leonzoane située dans la région sud du Mozambique (voir figure 1), cet article tente de mieux comprendre le caractère changeant des migrations de travail des familles depuis 1994 et la manière dont ces migrations constituent un élément central de recomposition des systèmes d'activités et du marché du travail rural.

Le cadre d'analyse est fondé sur l'approche SRL (sustainable rural livelihoods ou systèmes d'activités), adaptée pour mieux comprendre les activités familiales et leurs évolutions (Sourisseau et al., 2012). L’unité d'analyse définie pour cette étude est le système d'activités, conçu comme l'ensemble des actifs, capacités et activités des familles, tant au niveau local qu'en dehors de la communauté. Les activités de migration font ainsi partie d'une stratégie de systèmes d'activités multilocalisés. Le choix d'enquêtes biographiques et d'un échantillon stratifié (sous-zones) par quotas (groupes d'âge) auprès de quatre-vingt-dix-sept familles de Leonzoane a permis une analyse qualitative et quantitative représentative de la diversité des trajectoires de systèmes d'activités au cours des quinze dernières années. Dans cette perspective, la migration peut être vue comme une "transaction intertemporelle complexe entre le migrant et sa famille » (Guilmoto et Sandron, 2000). Cette définition permet d'observer les formes de circulations (Cortes et Faret, 2009), c'est-à-dire les flux et règles qui se créent ou se rompent entre les individus, les actifs et les ressources agricoles et non agricoles de la famille, au lieu d'origine et ailleurs, et éclaire les liens particuliers entre la migration et les différents capitaux (pris en compte dans l'approche SRL) et les modes familiaux d'organisation.

L'article est divisé en trois parties. La première partie resitue les enjeux du contexte de transition démo-économique mozambicain et de la région sud. La deuxième partie interroge les caractéristiques et le rôle des migrations économiques dans les stratégies des familles de Leonzoane au cours des quinze dernières années et les enjeux pour l'économie locale. Elle souligne la diversité des marchés du travail auxquels les familles participent et le rôle décisif des migrations de travail dans l'évolution des systèmes d'activités. À la lumière de ces résultats, la troisième partie questionne les fondements de la politique de développement rural incarnée par le Parp (Plan d'action pour la réduction de la pauvreté, 2011-2014) et propose une approche prenant mieux en compte les dimensions des migrations et de l'emploi rural. Il s'agit d'appuyer les stratégies d'investissement agricole, fondamentales pour la sécurité alimentaire, mais aussi d'identifier les effets positifs des circulations et migrations de travail sur le marché du travail rural. 


\section{CROISSANCE, PAUVRETÉ RURALE ET MIGRATIONS POSTAPARTHEID}

Initiant avec son adhésion au FMI en 1983 la fin de la période socialiste, le gouvernement mozambicain s'est engagé dans un processus de libéralisation économique. Après les accords de paix (1992), il a poursuivi les réformes de marché et démantelé les appareils de planification qui caractérisaient le pays jusqu'aux années 1990, tout en engageant une politique de privatisation et une transition à l'économie de marché. Le pays a également entamé un processus d'intégration commerciale avec l'entrée dans la Southern African Development Community (SADC) en 2008.

Les résultats de ces réorientations de politique économique ont été frappants : alors que le pays était un des plus pauvres du monde, des taux de croissance de $10 \%$ ont été enregistrés jusqu'au début des années 2000 (Kyle, 2003), puis de 7,5 \% par an en moyenne (GDM, 2011). Le pays est depuis lors référencé par la Banque mondiale et le FMI comme exemple de succès dans la mise en ouvre de ses politiques macroéconomiques.

Néanmoins, ce cadre macroéconomique ne se reflète pas dans l'amélioration du bien-être de la majorité des Mozambicains. Si le pays a connu des réductions des niveaux de pauvreté entre 1996 et 2002, l'essentiel de la population est restée sous le seuil de pauvreté. L'Enquête agricole nationale (INE, 2008) montre que la pauvreté et les inégalités continuent d'augmenter (Cunguara et Hanlon, 2010) avec écarts entre zones rurales et urbaines. Selon la troisième évaluation nationale de la pauvreté, son incidence est de 54,7 \% (GDM, 2010).

En effet, la croissance du Mozambique n'a pas donné lieu à une véritable transition économique. Malgré la progression des secteurs industriels et des services, $70 \%$ de la population mozambicaine vit en zone rurale (INE, 2009). Le secteur agricole est le secteur principal d'insertion de la population active, avec plus de $80 \%$ de la force de travail et, en 2010, $32 \%$ du PIB (World Bank, 2012). La croissance est liée à des " mégaprojets » largement appuyés par le stock d'IDE (investissements directs étrangers) qui a augmenté de façon fulgurante, de $29,1 \%$ du PIB en 2000 à $55 \%$ en 2010. Mais ces investissements concernent des activités intensives en capital avec une absorption limitée de travailleurs, notamment des non-qualifiés (Castel-Branco, 2008).

Cette lente transition économique est à mettre en parallèle avec la forte croissance de la population mozambicaine dont l'accompagnement institutionnel et en dotations physiques nécessaires pour garantir un niveau de vie décent est problématique. Même avec un accroissement des emplois salariés dans le secteur industriel, le pays ne peut pas créer des emplois au même rythme que l'accroissement de la population économiquement active (PEA), qui devrait plus que doubler d'ici à 2030 (World Bank, 2008). Ces éléments illustrent les limites de l'économie domestique et l'impasse dans laquelle se trouve le pays. 
Dans ce contexte, l'importance du travail salarié et des marchés du travail rural dans les stratégies des ménages ruraux au Mozambique a été mise en évidence au cours des quinze dernières années (Massingarela et al., 2005 ; Sender et al., 2006 ; Cramer et al., 2008). Dans les années 1980-1990, une abondante littérature a porté sur l'économie rurale mozambicaine, sur l'incidence et le rôle du travail salarié agricole, la croissance du phénomène migratoire dans l'articulation des économies de la région et le système de production colonial (voir entre autres Wuyts, 1981) ou encore sur les structures sociales en zones rurales et les formes de production différenciées (O'Laughlin, 1996). Si ces études offrent une vision de la réalité et de la complexité du marché du travail salarié et des stratégies de diversification des familles, la visibilité et la connaissance des activités de migrations au sein de ces stratégies restent encore très limitées (Cramer et Pontara, 1998). Elles ne permettent ni d'évaluer la place des activités de migration dans les stratégies, ni de comprendre les liens rural/urbain issus de la mobilité et leur rôle dans la diversification des activités des familles, ni comment les stratégies de différentes natures sont créées, reproduites ou érodées par la migration.

Or au regard de l'histoire nationale, la migration a été présente sous des formes changeantes pour les familles rurales et est un des facteurs importants dans les expériences de changement socioéconomique des zones rurales. Dès la période coloniale, une grande partie des familles rurales ont adapté leurs stratégies au système de plantations ou encore au système d'exportation de main-d'œuvre entre les Portugais et le régime sud-africain pour l'exploitation du secteur minier (1895-1975). Ainsi, les migrations de travail circulaires vers les régions minières sud-africaines étaient le choix de revenu privilégié des hommes. Ces migrations forcées ont mobilisé jusqu'à un tiers de la population active masculine dans la région sud (First, 1983) et sont à l'origine d'un imaginaire qui imprègne encore les pratiques des jeunes hommes. Après l'indépendance mozambicaine en 1975, la guerre civile (1977-1992) et l'échec des «villages communaux » en lien avec la stratégie rurale du gouvernement ont déplacé une large part de la population vers les villes. Certaines familles ont alors adopté des stratégies multirésidentielles et de diversification. Les travailleurs les mieux lotis restaient salariés dans le secteur minier ou entraient dans la nouvelle administration ; d'autres survivaient au gré d'activités informelles de l'économie de troc dominante (Mercandalli, 2013).

La fin de la guerre et la réinsertion de l'Afrique du Sud à l'économie régionale en 1994, doublées du relâchement des lois de contrôle des migrations par le régime d'apartheid, ont entraîné un regain de travailleurs mozambicains vers l'Afrique du Sud (Wa-Kabwe Segatti, 2008).

Par ailleurs, les migrations d'après-guerre sont complexes. En effet, les migrations internationales et internes, associées à de nouvelles motivations 
et opportunités encore mal comprises (Crush et McDonald, 2000), se sont intensifiées et modifiées (Black et al., 2006a). Outre l'intensité des mobilités, ce sont la diversité accrue de leurs formes et l'élargissement de la sphère sociospatiale et des liens rural/urbain des familles qui ressortent (Mercandalli, 2013). Sans nier l'existence de formes de réseaux (Newitt et Tornimbeni, 2008), on assiste à un renouvellement et un renforcement de ceux-ci (Ramos Cardoso et Maharaj, 2010) et de leurs effets sur l'organisation socioéconomique des familles (Lubkemann, 2009). Ainsi, la migration semble jouer un rôle crucial dans les moyens d'existence de milliers de familles (Black et al., 2006b).

\section{DIVERSITÉ DES FORMES D'AGRICULTURE FAMILIALE À LEONZOANE ET RÔLE DES MIGRATIONS}

Leonzoane (district de Massinga, dans la province d'Inhambane) appartient à la vaste plaine côtière sud-est du Mozambique (voir figure 1). Cette région semi-aride connait de fortes variations climatiques liées au système de mousson et présente des conditions agroécologiques moins favorables que les régions centre et nord du pays. L'agriculture représentative de la région sud est fondée sur l'élevage extensif et sur des cultures vivrières pluviales que l'on retrouve dans toute la province d'Inhambane selon un zonage est/ouest : maïs, haricot noir, arachide, manioc, noix de coco, noix de cajou. Toutefois, depuis que le secteur agricole mozambicain s'est ouvert à la concurrence internationale à partir des années 1990, les différenciations régionales se sont accentuées et, à Leonzoane, cela se traduit par un environnement institutionnel et un appui au secteur peu favorables en termes d'infrastructure, d'accès au crédit, de structures d'organisation et d'accès au marché.

\section{La diversité des stratégies des familles et le rôle différencié des migrations}

La typologie de trajectoires des systèmes d'activités entre 1994 et 2010 montre la diversité des stratégies des familles de Leonzoane, selon différents niveaux de diversification (agricole/extra-agricole) et de revenus (tableau 1). La mise en regard de chacune des stratégies avec les types de migrations et leurs caractéristiques (fréquence, destination, secteur, conditions d'emploi) montre combien leur rôle est décisif en tant qu'élément structurant des recompositions des stratégies des familles et source de différenciation économique.

Les sept types de trajectoires de systèmes d'activités sont présentés ici en quatre sous-ensembles soulignant le rôle des migrations dans les différenciations économiques à l'œuvre.

La stratégie " petite agriculture commerciale diversifiée et activité occasionnelle » (T1) rassemble les ménages avec une trajectoire stable depuis la fin de la guerre. Ces ménages combinent une petite agriculture commerciale diversifiée 
Tableau 1 : Typologie des sept types de trajectoires de systèmes d'activités et rôle des migrations (1994-2010)

\begin{tabular}{|c|c|c|}
\hline $\begin{array}{l}\text { Type de trajectoire } \\
\text { système d'activités }\end{array}$ & Type de migrations et rôle dans le système d'activités & $\begin{array}{c}\text { Type de } \\
\text { diversification }\end{array}$ \\
\hline $\begin{array}{l}\text { T1- Petite agriculture commerciale } \\
\text { diversifiée et activité extra-agricole } \\
\text { occasionnelle ( } 24 \%) \text { - \$ gpe } 4\end{array}$ & non migrants & - \\
\hline $\begin{array}{l}\text { T2- Alternance agriculture de } \\
\text { subsistance et salariat instable } \\
\text { (21\%) - \$ gpe } 4\end{array}$ & $\begin{array}{l}\text { Mobilités longues ou régulières multisite, sans règles } \\
\text { - ponction main-d'œuvre agricole } \\
\text { - transfert de compétences sans capital }\end{array}$ & $\begin{array}{l}\text { Extra-agricole } \\
\text { et agricole } \\
\text { occasionnelle }\end{array}$ \\
\hline $\begin{array}{l}\text { T3- Agriculture de subsistance puis } \\
\text { diversification extra-agricole ( } 21 \%) \\
\text { \$ gpe } 3\end{array}$ & $\begin{array}{l}\text { Mobilités fréquentes surtout nationales, règles liées au réseau local et } \\
\text { extérieur et au lignage } \\
\text { - accumulation non productive } \\
\text { - transfert de compétences et capital }\end{array}$ & $\begin{array}{l}\text { Extra-agricole } \\
(+/ \text { - agricole) }\end{array}$ \\
\hline $\begin{array}{c}\text { T4- Paysan-salarié (17 \%) } \\
\text { \$ gpe } 2\end{array}$ & $\begin{array}{l}\text { Mobilités régulières ou longues, internationales, } 1 \text { lieu, règles lignage et } \\
\text { réseaux familial / poste fixe } \\
\text { - accumulation non productive } \\
\text { - système de subsistance }\end{array}$ & stable \\
\hline $\begin{array}{l}\text { T5- Système transnational et } \\
\text { agriculture sous-exploitée (7 \%) } \\
\text { \$ gpe } 1\end{array}$ & $\begin{array}{l}\text { Mobilités très flexibles internationales / règles d'accès-contrôle des } \\
\text { ressources par polygamie hors lignage } \\
\text { - accumulation non productive/sécurité alimentaire } \\
\text { - transferts de compétences et capital, terre et/ou logement urbain }\end{array}$ & $\begin{array}{l}\text { stable } \\
\text { (extra-agricole) }\end{array}$ \\
\hline $\begin{array}{l}\text { T6- Migrant permanent avec } \\
\text { diversification agriculture } \\
\text { commerciale tardive }(4 \%) \$ \text { gpe } 2\end{array}$ & $\begin{array}{l}\text { Mobilité régulière, hybridation des règles réseaux \& lignage } \\
\text { - accumulation non productive / sécurité alimentaire }\end{array}$ & agricole \\
\hline $\begin{array}{c}\text { T7- Agriculture commerciale (6 \%) } \\
\text { \$ gpe 3-4 }\end{array}$ & $\begin{array}{l}\text { Migrants de retour/retraite } \\
\text { - accumulation non productive } \\
\text { - investissement productif agricole }\end{array}$ & \\
\hline
\end{tabular}

Source : Enquêtes 2009-2010.

en plus de leur système vivrier et complètent leurs revenus monétaires par du travail occasionnel comme salarié agricole et extra-agricole local (figure 2). Ils n’ont jamais migré et appartiennent au quatrième groupe de revenus le plus défavorisé (figure 3).

Dans les deux stratégies T2 et T3, les liens entre les activités de migration et le reste du système d'activités sont les plus importants. Les trajectoires montrent une diversification extra-agricole, avec parfois une diversification agricole plus tardive, mais avec des niveaux d'accumulation très différents.

Pour la stratégie " alternance agriculture de subsistance et salariat instable » (T2), les mobilités sont souvent liées à des activités informelles, surtout en Afrique du Sud, dans les services, la construction ou les mines. Elles ne donnent quasiment pas lieu à des flux d'échanges (monétaires ou matériels) ni à des règles associées à l'usage des bénéfices de la migration. Les ménages sont pris dans des trappes à pauvreté, l'activité de migration fragilise le système vivrier sans consolidation des ressources des activités extérieures. Leurs revenus irréguliers varient selon le moment de leur trajectoire (figure 3).

Pour la stratégie de type «agriculture de subsistance puis diversification extra-agricole » (T3), les familles se différencient par un usage décisif des réseaux acquis pendant la guerre, qui permet un accès continu à une activité formelle 
ou informelle et l'engagement des femmes dans des activités extra-agricoles locales. Après une phase d'installation de la famille, elles entrent dans des processus d'accumulation et diversifient leurs activités localement (figure 2). Leurs mobilités sont variées mais plutôt nationales. Elles sont associées, d'une part, à des transferts de compétences et de capital et, d'autre part, à des règles de la migration souvent liées aux autorités traditionnelles du lignage pour le mariage et l'accès à la terre. Ces migrations sont aussi associées au réseau familial ou professionnel pour l'accès à des ressources extérieures (information, emploi, logement...) et pour la gestion des actifs du patrimoine familial.

Un troisième sous-ensemble regroupe des stratégies qui présentent des trajectoires stables, T4 et T5. Elles combinent agriculture de subsistance et un emploi formel dans le secteur minier ou ailleurs. La migration n'a pas d'effet de diversification, mais elle est fondamentale pour la reproduction du système d'activités familial, selon deux logiques très différentes.

Pour les stratégies de type "paysan-salarié » (T4), les mobilités sont essentiellement internationales, vers l'Afrique du Sud, de longue durée et vers un seul lieu. Elles sont associées à des règles de transmission de postes stables du secteur formel (mine ou autre) au sein du lignage. Après l'installation de la famille, les bénéfices de la migration servent à renforcement du système vivrier (main-d'œuvre, bovins, stockage) et à de l'accumulation non productive (habitat, biens durables, santé, éducation).

Pour les stratégies de type "système transnational avec agriculture sousexploitée » (T5), les familles se distinguent par leur résidence urbaine pendant la guerre et une faible dotation en terre. Elles ont les mobilités les plus flexibles dans plusieurs villes de l'espace national et international. La plupart ont commencé comme salariés informels dans les secteurs des services ou de la construction, puis sont parvenus à créer une ou plusieurs activités indépendantes grâce à leur réseau. Les revenus de la migration servent à l'installation de la famille avec une double résidence et à sa sécurité alimentaire. L'agriculture est marginale ou inexistante et la terre sert de patrimoine d'assurance en cas de choc. Les familles contrôlent leurs ressources à Leonzoane et en Afrique du Sud via la multirésidence tournante grâce à leur statut polygame. Ces deux systèmes d'activités très stables (T4 et $\mathrm{T} 5$ ) répondent aux groupes de revenus les plus élevés (figure 3), mais sont très dépendants de la migration et exposés en cas de crise.

Enfin, dans les deux stratégies T6 et T7, le système d'activités évolue vers une diversification agricole commerciale dans la deuxième ou troisième phase du cycle de vie du ménage. Pour les stratégies de type «migrants permanents avec diversification agricole commerciale tardive "(T6), les migrants sont des réfugiés nationaux, restés en ville à la fin de la guerre avec un emploi stable. Ils ont néanmoins maintenu des liens forts avec leur famille et décident 
Figure 2 : Stratégies des familles : principales combinaisons d'activités (1994-2010)

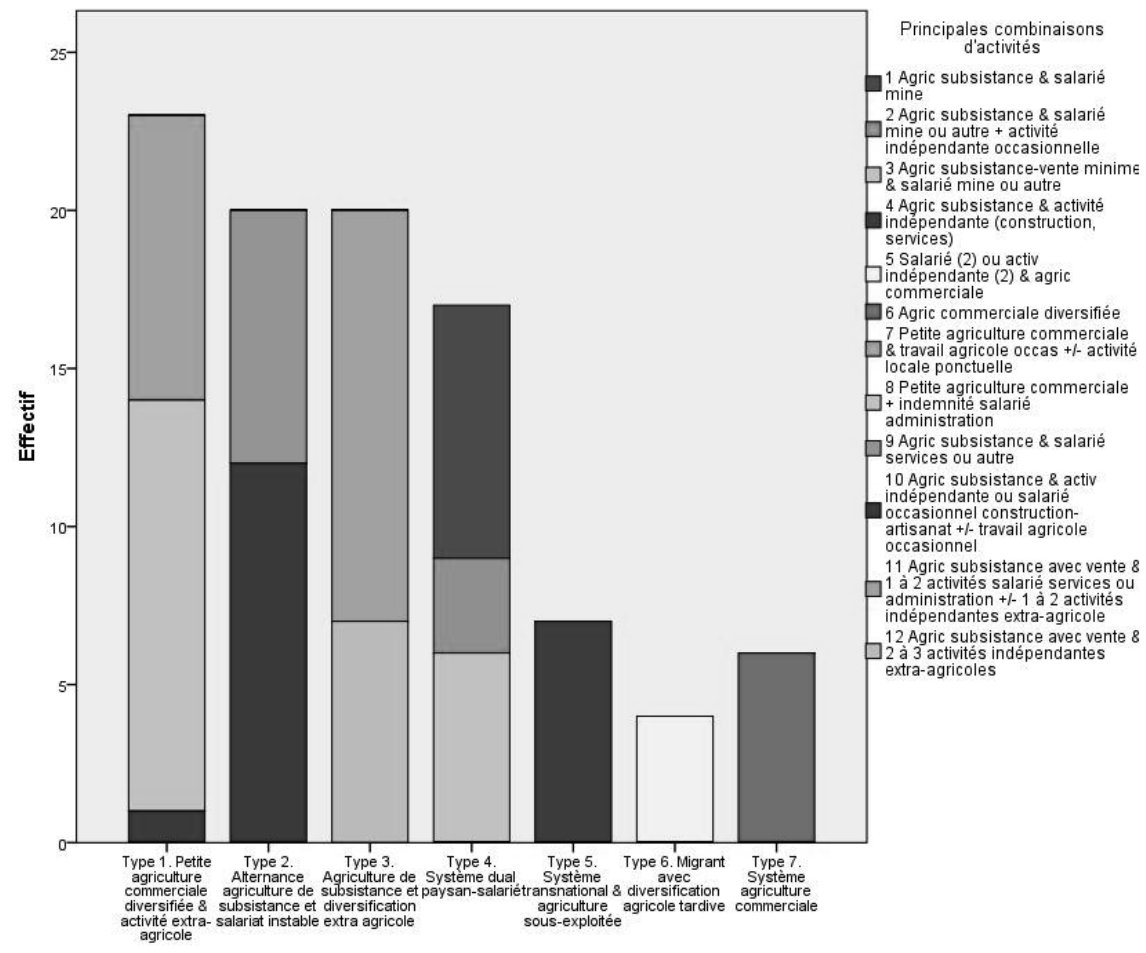

Source : Mercandalli, 2013.

d'investir dans l'agriculture commerciale sur les terres familiales pour compléter leurs revenus urbains ou diminuer leur budget alimentaire. Dans les stratégies fondées sur l'" agriculture commerciale »(T7), les bénéfices de la migration sont utilisés au moment qui correspond à la retraite, pour investir dans l'agriculture commerciale locale, dans l'élevage bovin pour la location/vente ou dans des cultures de rente (figure 1). Cela permet de maintenir des revenus proches du niveau du salaire minimum national (figure 3 )

L'ensemble de ces stratégies montrent d'une part la diversité des migrations des familles inscrites dans des logiques de circulation et d'échanges rural/urbain où les réseaux ont un rôle central et, d'autre part, l'émergence décisive d'arrangements intra- et interfamiliaux liés aux migrations quant à l'accumulation et à la différenciation rurale à Leonzoane.

En lien avec ces caractéristiques des mobilités du contexte postapartheid, les trajectoires professionnelles permettent d'estimer que la part du temps de travail des activités de migration représente en moyenne $40 \%$ au niveau du ménage contre $25 \%$ au cours de la période coloniale sous le système 
Figure 3 : Distribution des types de systèmes d'activités selon le niveau de revenu en metical/mois (2009-2010, Leonzoane, 97 familles enquêtées)

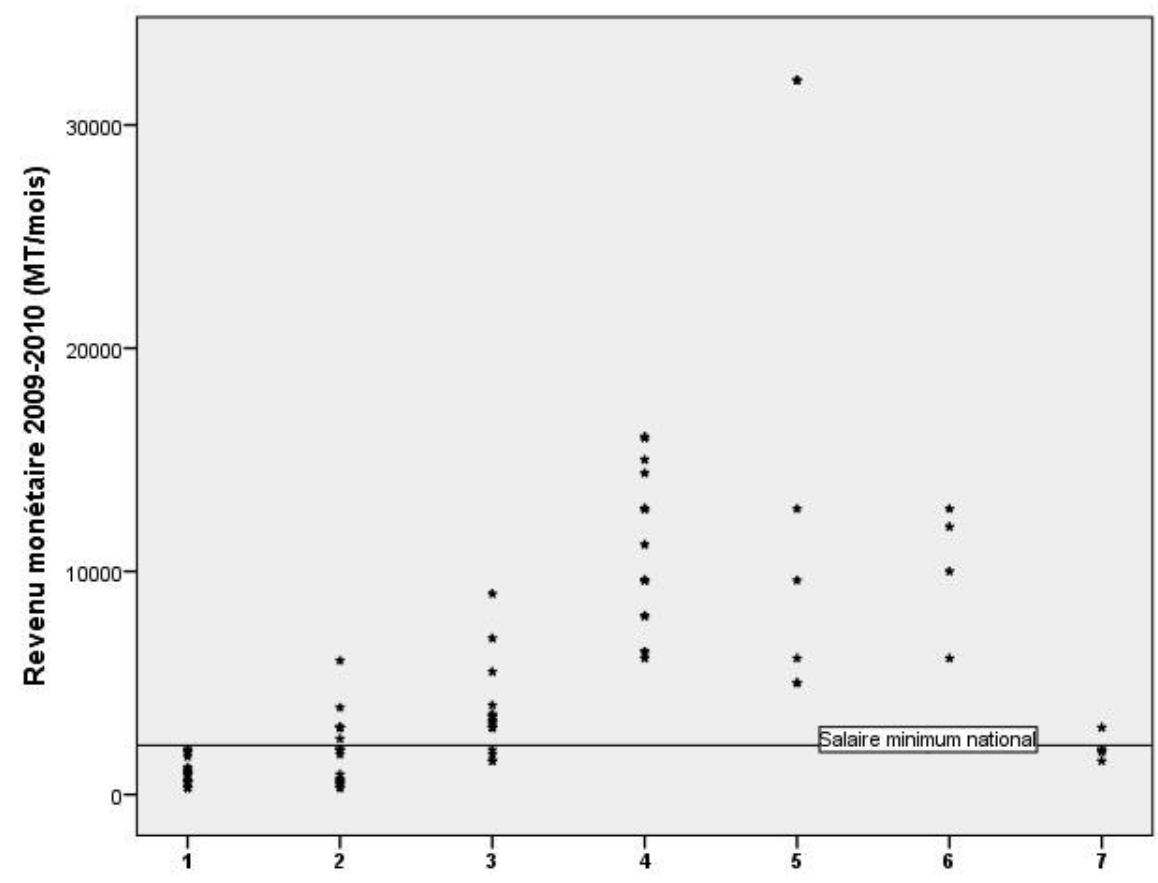

Type système d'activités et stratégie, période économie de marché

Sources : Mercandalli, 2013.

d'exportation de main-d'œuvre. Cela montre le poids des activités de migration dans la composition structurelle du travail des ménages de Leonzoane. Enfin, les résultats soulignent aussi la diversité des marchés du travail à la fois rural et extérieur dans lesquels la majorité des familles sont insérées (70 \% des familles), associant espaces rural et urbain au sein de systèmes familiaux multilocalisés.

\section{Économie de dépendance vis-à-vis des migrations, segmentation du marché du travail rural et système économique local instable}

Les analyses à l'échelle de Leonzoane indiquent que les migrations de travail sont source de changements de l'économie locale à trois niveaux.

Tout d'abord, les ressources de la migration servent en priorité à l'investissement non productif pour l'habitat et la consommation de biens durables (73\% des familles), à l'appui au système agricole de subsistance (54\%) et à l'accès à la terre via le financement du mariage (40\%). Ensuite, une partie des familles mobilise les revenus de la migration en investissement productif extra-agricole (30\% des familles) ou agricole (14\%). De plus, les revenus de la migration servent aussi à la sécurité alimentaire de la famille (20\% des familles). Ainsi, le 
principal mécanisme liant l'économie locale à la migration est un mécanisme de reproduction et de dépendance économique, plus que de création de richesses.

Lorsque la migration sert à l'investissement productif (stratégies T3, T5, T6, T7), $75 \%$ des familles l'emploient à des activités extra-agricoles. Ainsi, l'accroissement des activités extra-agricoles et la diminution relative de la place de l'agriculture (reproduction des structures à l'identique ou accroissement minime pour une minorité) indiquent une tendance à la désagrarisation. Cela confirme le poids de la migration dans les stratégies des familles rurales en Afrique subsaharienne (Black et al., 2006b), en lien avec les processus de diversification et de désagrarisation (Bryceson, 2005).

Le deuxième niveau de transformation est visible dans les recompositions du marché du travail rural. L'insertion d'une partie des familles sur le marché du travail extérieur tend à modifier les relations de production entre familles. Cela souligne la concurrence entre les incitations salariales locales et extérieures d'une part, agricoles et non agricoles d'autre part. Les recompositions du marché du travail local peuvent être appréhendées au travers des relations de travail entre les différentes stratégies des familles de Leonzoane, influencées par les formes du rapport salarial, notamment entre migrants et non-migrants. Ainsi, les stratégies locales « petite agriculture commerciale diversifiée » (T1) et " alternance agriculture de subsistance et salariat instable » (T2) regroupent l'essentiel des familles de non-migrants ou de migrants de retour. Or, ce sont les seules à s'engager sur le marché du travail agricole local, pour compléter leurs faibles revenus monétaires, notamment au moment du pic crucial de travail du labour/semis. En plus de leurs propres parcelles, ces familles répondent à la demande de main-d'œuvre des stratégies " agriculture commerciale » (T7), « paysan-salarié » (T4), « systèmes transnationaux » (T5) et "diversification agriculture commerciale tardive » (T6), engagées sur le marché national et international et qui les rémunèrent avec leurs revenus extérieurs. Cette configuration souligne des tensions au sein du marché du travail rural au détriment du marché agricole. En effet, les systèmes de coopération et d'échange de main-d'œuvre diminuent, et le paiement en espèce (ou en biens) se généralise, surtout avec les revenus de la migration, ainsi que le renchérissement du coût de la main-d'œuvre agricole. Cela fragilise les stratégies locales fondées sur l'agriculture $(\mathrm{T} 1, \mathrm{~T} 2)$ avec des revenus monétaires irréguliers ou sans accès à l'entraide locale.

Un troisième niveau d'articulation existe entre les stratégies les plus aisées fondées sur les activités de migration (T4, T5, T6), et les stratégies locales « agriculture puis diversification extra-agricole » (T3) et celles prises dans des trappes à pauvreté " alternance agriculture de subsistance et salariat instable » (T2). Ces deux stratégies dépendent en partie de la demande de biens et services des migrants pour le développement de leurs activités extra-agricoles locales ou 
leurs activités occasionnelles (construction, réparations, gardiennage et autres services, consommation lors des retours).

L'ensemble de ces éléments souligne l'importance des migrations de travail dans le processus de formation et de segmentation du marché du travail rural, agricole et non agricole, de par les articulations qu'elles créent entre les différentes stratégies des familles et leurs principaux secteurs d'insertion. Elles sont ainsi un élément structurant du fonctionnement du marché du travail local. Cela confirme par ailleurs l'importance du travail salarié et la diversité du marché du travail rural informel et du « biscato ${ }^{1}$ » dans les stratégies des familles (Oya, 2010), tout en précisant le rôle déterminant des migrations de travail dans ces processus. Le degré et les formes de segmentation du marché du travail rural $\mathrm{local}^{2}$ et extérieur dans lesquels sont insérées les familles, par zone géographique, sous-secteur, type d'emploi, sont un résultat important. Les différences entre les formes de travail salarié, relatives aux conditions de travail, à la fréquence, aux modalités et niveaux de rémunération, signalent des processus différents, d'une part de reproduction de situations de pauvreté extrême (par exemple T2), et d'autre part de formes de sortie de la pauvreté (par exemple T3, T6).

\section{Prospective : vers plus de flexibilité et de vulnérabilité des stratégies des familles?}

L'évolution des stratégies par groupe d'âge (tableau 2) donne une indication des dynamiques et de la tendance à la croissance, à la stabilité ou à la diminution de ces stratégies d'une génération à l'autre - tout en tenant compte des effets de cycle de vie. Trois grandes tendances ressortent.

Premièrement, le "système dual renouvelé du paysan-salarié » (T4), semble s'amenuiser (17\% des familles d'aujourd'hui). Cette stratégie, associée à des migrations longues et stables avec des conditions d'emploi formelles, ne concerne plus que $27 \%$ des 20-40 ans et $23 \%$ de leurs aînés actifs de 40-60 ans. La diminution de cette stratégie, liée à une ressource circulatoire autour de rentes de postes fixes, est concomitante de la rationalisation croissante de ces emplois, souvent dans le secteur minier sud-africain, et associés à des compétences précises.

Deuxièmement, les stratégies "agriculture et salariat instable»(T2) et " agriculture de subsistance puis diversification » (T3) sont les plus répandues aujourd'hui (20,6\% chacune), en particulier chez les jeunes. La stratégie T2

1. « Biscato » ou « ganho-ganho » : travail salarié occasionnel rémunéré en monnaie ou en nature, très commun au Mozambique et dans d'autres pays de la région (Whiteside, 2000).

2. Parmi les petits emplois informels courants, on trouve la transformation et la vente de fruits ou céréales en boissons et eaux-de-vie (femmes), l'extraction/vente de bois et de pierres, une variété de travaux agricoles ainsi que la location de bœufs, divers métiers de la construction (maçons, charpentiers, etc.), ou de la confection comme couturiers (uniformes scolaires). Dans le secteur formel, on trouve des emplois comme agent éducatif ou de santé ou représentant administratif. 
Tableau 2 : Distribution des groupes d'âge des familles par type de stratégies (1994-2010)

\begin{tabular}{|c|c|c|c|c|c|c|}
\hline & & & \multicolumn{4}{|c|}{ Groupe d'Age par tranches de 20 ans } \\
\hline & & & $20-40$ & $41-60$ & $60+$ & Total \\
\hline \multirow{15}{*}{$\begin{array}{l}\text { Type système } \\
\text { d'activité et } \\
\text { stratégie période } \\
\text { éco de marché }\end{array}$} & \multirow{2}{*}{$\begin{array}{l}\text { Type } 1 \text { Petite agriculture commerciale } \\
\text { diversifiée \& activité extra agricole }\end{array}$} & Effectif & 1 & 10 & 12 & 23 \\
\hline & & $\mathrm{N} \%$ colonne & $3,3 \%$ & $25,6 \%$ & $42,9 \%$ & $23,7 \%$ \\
\hline & \multirow{2}{*}{$\begin{array}{l}\text { Type } 2 \text { Alternance agriculture de subsistance } \\
\text { et salariat instable }\end{array}$} & Effectif & 10 & 5 & 5 & 20 \\
\hline & & $\mathrm{N} \%$ colonne & $33,3 \%$ & $12,8 \%$ & $17,9 \%$ & $20,6 \%$ \\
\hline & \multirow{2}{*}{$\begin{array}{l}\text { Type } 3 \text { Agriculture de subs istance et } \\
\text { divers ification extra agricole }\end{array}$} & Effectif & 5 & 9 & 6 & 20 \\
\hline & & $\mathrm{N} \%$ colonne & $16,7 \%$ & $23,1 \%$ & $21,4 \%$ & $20,6 \%$ \\
\hline & \multirow[t]{2}{*}{ Type 4 Système Dual renouvelé } & Effectif & 8 & 9 & 0 & 17 \\
\hline & & $\mathrm{N} \%$ colonne & $26,7 \%$ & $23,1 \%$ & $0,0 \%$ & $17,5 \%$ \\
\hline & \multirow{2}{*}{$\begin{array}{l}\text { Type } 5 \text { Système Transnational\&agriculture ss } \\
\text { exploitée }\end{array}$} & Effectif & 4 & 2 & 1 & 7 \\
\hline & & $\mathrm{N} \%$ colonne & $13,3 \%$ & $5,1 \%$ & $3,6 \%$ & $7,2 \%$ \\
\hline & \multirow{2}{*}{$\begin{array}{l}\text { Type } 6 \text { Migrant avec diversification agricole } \\
\text { tardive }\end{array}$} & Effectif & 2 & 2 & 0 & 4 \\
\hline & & $\mathrm{N} \%$ colonne & $6,7 \%$ & $5,1 \%$ & $0,0 \%$ & $4,1 \%$ \\
\hline & \multirow[t]{2}{*}{ Type 7 Systeme agriculture commerciale } & Effectif & 0 & 2 & 4 & 6 \\
\hline & & $\mathrm{N} \%$ colonne & $0,0 \%$ & $5,1 \%$ & $14,3 \%$ & $6,2 \%$ \\
\hline & Total & $\mathrm{N}$ total & 30 & 39 & 28 & 97 \\
\hline
\end{tabular}

est la plus courante pour les 20-40 ans (33\%) et concerne moins de 40-60 ans, qui ont plus d'expérience et qui ont potentiellement réussi à basculer vers une diversification de type T3. Ces deux stratégies sont liées à une variété de migrations incluant des profils émergents très mobiles. Elles correspondent à des emplois aux niveaux national et international, davantage informels et sous des statuts variés. Ces emplois sont les plus propices à l'acquisition et aux transferts de connaissances. Au regard de la croissance démographique, ces deux stratégies, qui totalisent $41,2 \%$ des familles enquêtées, constituent un point central de la problématique du devenir de l'économie locale, d'autant plus qu'elles sont liées aux systèmes d'activités les plus flexibles et avec le plus d'interactions avec les activités de migration. L'expansion du type T3 représenterait une fenêtre pour développer des effets d'entraînement locaux, alors que celle du type T2 signifierait une fragilisation des systèmes d'activités des nouveaux actifs entrants, via l'expansion d'emplois occasionnels en conditions précaires. Situées entre les deux extrêmes, d'un côté les stratégies agricoles locales des plus démunis (T1) et de l'autre des stratégies duales (T4) dépendant des revenus de la migration, contrecarrant le processus de polarisation. Ces deux stratégies $\mathrm{T} 2$ et T3 forment un noyau relevant d'un système idéal-typique de résilience susceptible de représenter une troisième voie.

Troisièmement, l'émergence au sein des générations 20-40 ans et 41-60 ans des systèmes « transnational et agriculture sous exploitée » (T5) et « migrant et diversification agricole tardive » (T6), incarnant de nouveaux modes d'organisations liés à la migration, peut aussi peser sur les dynamiques locales à venir. Ces stratégies aujourd'hui minoritaires offrent aussi des voies de développement potentiel pour l'économie locale dans le secteur agricole. 
Finalement, ces éléments soulignent l'intérêt de discerner les logiques et les fonctionnements très différents des stratégies en lien avec la migration pour être en mesure de proposer des orientations adaptées, favorisant un développement inclusif plutôt qu'un système d'économie de dépendance avec un équilibre $a$ priori fragile et un risque d'instabilité.

\section{RÉFLEXIONS POUR UNE POLITIQUE INTÉGRÉE DE DÉVELOPPEMENT RURAL}

Les résultats du travail mené à Leonzoane questionnent les fondements de la stratégie nationale de lutte contre la pauvreté et le développement rural : dans quelle mesure les objectifs de la stratégie nationale de développement rural, incarnée par le Poverty Reduction Action Plan (PARP) (2011-2014), prennent-ils en compte ces recompositions de l'agriculture familiale en lien avec les migrations de travail et l'emploi salarié ?

Le gouvernement mozambicain a pour principal objectif de gérer une croissance économique inclusive et de réduire les niveaux d'incidence de pauvreté et de vulnérabilité du pays (PARP 2011-2014) (GDM, 2011). Pour cela, les secteurs productifs de l'agriculture et de la pêche ont été mis en avant, du fait du grand nombre de familles pauvres vivant directement ou indirectement de l'agriculture (Oya, 2012) ${ }^{3}$. Deux angles sont privilégiés. D’une part, la production de produits alimentaires de base à l'usage de la population pour lutte contre la faim. Ici, un rôle fondamental est attribué à la production du secteur familial de petite taille, souvent considéré comme secteur de subsistance. D'autre part, la création d'emploi à travers le développement d'une grande agriculture commerciale tournée vers l'exportation, fondée sur l'attraction d'investisseurs étrangers et nationaux. Avec ce mécanisme, des dynamiques de création d'emploi salarié et de revenus sont attendues à partir de la création d'entreprises agricoles et de l'émergence de producteurs locaux, d'associations de producteurs ou de contract farming.

Au-delà des problèmes d'une vision dualiste selon laquelle l'économie rurale mozambicaine est divisée en deux secteurs, cette politique ignore l'importance pour les familles des formes de travail (salarié et indépendant) local ou extérieur. Ainsi, le PARP ne saisit pas les dynamiques de production et d'accumulation actuelles et perd la possibilité de mettre à profit ces dynamiques pour mobiliser des ressources financières et développer des effets économiques spécifiques.

3. Différents documents de politiques nationales et mesures de politiques agricoles sont mis en œuvre : PEDSA (Plano Estratégico para o Desemvolvimento do Sector Agrario), PNISA (Plano Nacional de Investimento do Sector Agrario), PACTO pour le développement du secteur agraire au Mozambique dans le contexte du « Programme détaillé de développement de l'agriculture africaine » (CAADP). 
L'étude du cas de Leonzoane a en effet mis en évidence que ce sont surtout les dynamiques des migrations de travail et des marchés du travail rural, et leurs articulations, qui expliquent les processus d'accumulation, y compris pour l'agriculture dont la place dans les stratégies des familles reste assez stable. La nature des supports politiques serait alors mieux dirigée vers des leviers qui ont généralement des effets bénéfiques sur tous les types d'activités économiques en zone rurale et sur les circulations rural/urbain plutôt que d'être centrée sur l'agriculture seule.

Une telle approche peut être illustrée à partir de l'expérience de Leonzoane. La voie de développement de l'économie locale implique de développer les stratégies d'investissement agricole, fondamentales pour la sécurité alimentaire, et de soutenir les effets bénéfiques du travail salarié et des circulations, pour renforcer le marché du travail rural. Pour cela, il est important d'identifier les groupes de population qui requièrent une attention particulière, avec des caractéristiques et des mécanismes de sortie de la pauvreté qui diffèrent. Dans ce sens, la stratégie de production familiale "agriculture de subsistance et diversification extra-agricole» (T3) pourrait constituer un groupe d'intérêt pour la formulation de mesures ciblées favorisant des effets d'entraînement sur l'économie locale, participant au développement du marché du travail local non agricole et agricole, en complémentarité avec les autres stratégies de Leonzoane (Mercandalli, 2014). Par ailleurs, les familles qui travaillent pour d'autres en zone rurale ou en ville ne font pas toutes partie des plus pauvres, mais les plus pauvres ont tendance à pratiquer des formes de travail salarié précaires, rémunérées en espèce ou en nature. Pour ces familles, le renforcement des marchés du travail rural peut suivre trois axes : d'abord, la promotion de cultures plus intensives en travail (horticulture par exemple), qui mènent également souvent à une hausse de la proportion d'emplois saisonniers réguliers et plus stables, contrairement aux emplois occasionnels ; ensuite, des mesures promouvant des accroissements de productivité qui peuvent être transformés en salaires ruraux plus élevés; et pour finir, l'appui à l'industrialisation rurale et à la création de relations entre secteurs agricole et non agricole. Enfin, les stratégies des familles fondées sur la migration nationale et internationale et associée à différents secteurs et statuts d'emploi invitent à identifier et à lever les contraintes publiques et privées qui pèsent sur ces stratégies, à promouvoir et adapter des mécanismes de mobilités sectorielle et géographique par des canaux légaux et sûrs.

Cela conduit à une seconde vision de l'économie rurale, divergente de celle $\mathrm{du}$ gouvernement mozambicain. Cette vision, également mise en avant par d'autres recherches au Mozambique (Oya, 2010), suggère que l'économie rurale est liée organiquement et structurellement aux marchés du travail nationaux et régionaux, centraux pour les besoins d'un grand nombre de ruraux africains pauvres. Cette conception reconnaît que la pluriactivité, l'emploi informel et 
de formes précaires, ainsi que l'accroissement des liens rural/urbain et des migrations modifient les structures et le fonctionnement des économies rurales du fait des interactions qu'ils créent entre les stratégies des ménages et le système économique local et national.

Cette vision remet en cause les fondements du PARP et le lien direct entre agriculture et réduction de la pauvreté. Cela appelle à sa réorientation afin, d'une part, de promouvoir l'emploi comme élément central de la stratégie de développement à moyen terme et, d'autre part, de mettre en place un cadre politique qui permette que la création d'emploi productif et le développement économique aient lieu simultanément.

Cette forme de réorientation requiert la formulation de politiques intégrées de développement rural permettant de prendre en compte les dynamiques intersectorielles et spatiales à l'œuvre. Cette approche intégrée de politiques de développement et leur durabilité semblent dépendre de plusieurs facteurs. Elles dépendent d'abord de mesures facilitant la mobilité du travail, telles que des infrastructures de transport et des informations sur les opportunités de travail dans les différentes parties de l'économie pour diminuer les barrières à l'entrée et atténuer certains des aspects inégalitaires des migrations rurales. Elles dépendent aussi de l'intégration progressive des activités locales au secteur formel (structuration des secteurs clés et amélioration des conditions d'entrée). Elles dépendent enfin de la stabilité d'une demande locale liée aux stratégies familiales davantage fondées sur l'agriculture. Plus particulièrement, cette approche nécessiterait la cohérence des processus de formulation du PARP et de la Stratégie d'emploi et de formation professionnelle (GDM, 2006) selon les secteurs les plus dynamiques et groupes cibles les plus vulnérables, ainsi que la cohérence des différentes stratégies sectorielles nationales qui sont aujourd'hui formulées de façon autonome vis-à-vis du PARP (Castel-Branco, 2012). Enfin, une bonne gouvernance des migrations devrait concilier les priorités à court et à long terme, mais aussi les intérêts, les besoins et les droits des migrants, ainsi que des pays d'origine et de destination. Cette gouvernance passe par le renforcement des capacités d'harmoniser la politique migratoire avec les priorités des autres domaines d'action publique; des capacités et moyens d'action favorisant des formes de mobilité circulaires et temporaires; des approches centrées sur les migrants, axées sur les droits, les compétences et le capital humain (OIM, 2012).

Les orientations proposées ici sont générales. Ce qui importe, c'est qu'un cadre politique intégré reconnaissant ces besoins de politique soit mis en place et hiérarchise les interventions qui favorisent les conditions pour la création plus rapide d'emploi rural, indépendant et salarié, de façon adaptée aux différents contextes régionaux du pays. 


\section{CONCLUSION}

Cette recherche conduite dans la région sud du Mozambique a mis en évidence différents types de migrations et formes de circulations dans les stratégies des familles de Leonzoane. Elle a montré comment les migrations de la période actuelle sont un élément structurant et déterminant des recompositions des systèmes d'activités et du marché du travail rural, source de différenciation économique. Les formes de migration et les conditions de travail des migrants depuis la fin de l'apartheid sont ainsi devenues plus variées. Elles sont associées à une diversité de stratégies et à un plus fort degré de différenciation que ce qui prévalait depuis le milieu du XIX ${ }^{\mathrm{e}}$ siècle jusqu'aux années 1990.

Au-delà de la région sud du Mozambique, la migration interne ou internationale est une stratégie décisive pour des milliers de familles rurales en Afrique australe, mais cette réalité est rarement reconnue par les politiques de réduction de la pauvreté (Black et al., 2006b). Le caractère répandu des formes d'agricultures familiales en partie fondées sur la pluriactivité et multilocalisées, en Afrique australe (notamment les anciennes « réserves de travail ») et dans d'autres régions du monde, souligne l'importance sous-estimée des liens rural/urbain dans les processus de restructuration économique, comme un champ à explorer pour les politiques publiques (Tacoli, 2002).

Les migrations de travail, les liens rural/urbain et la diversification des activités des familles rurales changent ainsi la donne des connaissances conventionnelles du processus de restructuration et de développement. Il semble que le devenir de Leonzoane ne passe ni par l'offre de travail illimitée du secteur agricole ni par le processus linéaire de transition du secteur primaire vers les secteurs industriel et tertiaire. Il n'y aurait ainsi pas de sens unilinéaire de la transition et du processus de développement.

\section{BIBLIOGRAPHIE}

Black R., J. Crush et al., 2006a, " African Migration and development series ", Migration and Development in Africa. An Overview, Cape Town : Southern African Migration Project.

Black R., Natali C. et al., 2006b, " Migration and inequality », Equity and Development, rapport sur le développement mondial 2006, document de travail.

Bryceson D. F., 2005, « Rural livelihoods and agrarian change in sub-Saharan Africa, processes and policies ", in Ellis F. (dir.), Rural Liveli- hoods and Poverty Reduction Policies, New York : Routledge, pp. 48-61.

Castel-Branco C., 2008, Desafios do desenvolvimento rural em Moçambique. Contributo crítico com debate de postulados básicos, Colecção de discussion papers do IESE, Maputo : IESE.

Castel-Branco C., 2012, "PARP 2011-2014. Contradiçoes, tensoes e dilemas ", in de Brito Luis et al. (dir.), Desafios para Moçambique 2012, Maputo : IESE, pp. 105-115. 
Cortes G. et Faret L., 2009, Les Circulations transnationales. Lire les turbulences migratoires contemporaines, Paris : Armand Colin.

Cramer C., Carlos 0. et al., 2008, Rural Labour Markets in Sub-Saharan Africa. A New View of Poverty, Power and Policy, Centre for Development Policy and Research, Policy briefs, $n^{\circ} 1$, novembre.

Cramer C. et Pontara N., 1998, "Rural poverty and poverty alleviation in Mozambique. What's missing from the debate? ", The Journal of Modern African Studies, vol. 36, no 1 , pp. 101-138.

Crush J. et McDonald D. A. (dir.), 2000, Transnationalism, African Immigration and New Migrant Spaces in South Africa, numéro spécial du Canadian Journal of African Studies, vol. 34, n० 1, pp. 1-19.

Cungara B., Fagilde G. et al., 2012, «Growth without change ? À case study of economic transformation in Mozambique ", Journal of African Development, vol. 14, $n^{\circ} 2$.

Cunguara B. et Hanlon J., 2010, « Poverty is not being reduced in Mozambique ", Crisis States Working Papers, $n^{\circ} 74$.

De Vletter F., 2007, « Migration and development in Mozambique. Poverty, inequality and survival », Development Southern Africa, vol. 24, $n^{\circ} 1$, p. 18.

First R., 1983, Black Gold. The Mozambican Miner, Proletarian and Peasant, New York : Palgrave Macmillan.

GDM, 2006, "Estratégia de emprego e formação profisional 2006-2015 », ministère du Travail, Maputo.

GDM, 2010, " Pobreza e bem estar em Moçambique. Terceira avliação nacional ", ministère de la Planification et du Développement, Maputo.

GDM, 2011, " Plano de acção para redução da pobreza (PARP) 2011-2014 ", Maputo.

Guilmoto C. Z. et Sandron F., 2000, "La dynamique interne des réseaux migratoires dans les pays en développement », Population and
Development Review, vol. 55, n 1 , pp. 105134.

INE, 2008, "TIA, Trabalho de Inquérito Agrícola ", ministère de l'Agriculture, Maputo : INE.

INE, 2009, Censo da População 2007. Maputo : INE.

Kyle S., 2003, " Political and economic prospects for Mozambique and Angola ", document de travail, département d'économie appliquée et de management, Cornell University, Ithaca : New York.

Lubkemann S. C., 2009, « From circular migrants in the mines to transnational polygynists in the townships. A century of transformation in central mozambican male migration regimes (1900-1999) ", International Migration, numéro spécial sur les migrations dans le monde lusophone, vol. 47, n 3, pp. 51-92.

Massingarela C., Virgulino N. et al., 2005, « Mercados rurais de emprego em Moçambique. Um estudo sobre 0 trabalho assalariado temporario e informal nas zonas rurais de Manica, Nampula e Zambezia », ministère de la Planification et du Développement, documents de travail.

Mercandalli S., 2014 (à paraître), "L'agriculture dans le sud du Mozambique, une activité fondée sur les migrations de travail », in Diversité des agricultures familiales , ouvrage collectif, Versailles : Éditions Quae.

Mercandalli S., 2013, Le Rôle complexe des migrations dans les reconfigurations des systèmes d'activités des familles rurales : la circulation comme ressource? Localité de Leonzoane, Mozambique 1900-2010, thèse de doctorat en sciences économiques, université ParisSud-XI.

Newitt M. et Tornimbeni C., 2008, "Transnational networks and internal divisions in central Mozambique. An historical perspective from the colonial period ", Cahiers d'études africaines, pp. 707-740. 
OIM, 2012, " Cycles économiques, évolution démographique et migration », Rapport international sur la migration.

O'Laughlin B., 1996, "Through a dividing class. Dualism, class and the agrarian question in Mozambique », Journal of Peasant Studies, vol. 23, $n^{\circ} 4$, pp. 1-39.

Oya C., 2010, "Rural inequality, wage employment and labour market formation in Africa. Historical and micro-level evidence ", document de travail.

Oya C., 2012, " Crise Global, Crescimento e desafios para Moçambique e sua estratégia de desenvolvimento », in de Brito Luis et al. (dir.), Desafios para Moçambique 2012, Maputo: IESE, pp. 387-409.

Pendleton W., Crush J. et al. (dir.), 2006, Migration, Remittances and Development in Southern Africa, Cape Town : Insitute for Democraty in South Africa/Southern African Migration Project.

Ramos Cardoso M., Maharaj B. et Preston-Whyte E., 2010, «Social networks and undocumented Mozambican migration to South Africa ", Geoforum, vol. 41, n 6 , pp. 885-896.

Sender J., Oya C. et al., 2006, " Lifting the blinkers. A new view of power, diversity and poverty in mozambican rural labour markets », Journal of Modern African Studies, vol. 46, $n^{\circ} 3$, pp. 361-392.

Sourisseau J. M., Bosc P. M. etal., 2012, " Représenter la diversité des formes familiales de la production agricole. Approches théoriques et empiriques », Montpellier : document de travail ART-Dev 2012.

Tacoli C., 2002, " Changing rural-urban interactions in sub-Saharan Africa and their impact on livelihoods. A summary », document de travail, travaux sur les interactions zones rurales/urbaines et les stratégies de subsistance.

Wa Kabwe-Segatti A., 2008, "Reforming South African immigration policy in the post-apartheid period (1990-2006): what it means and what it takes ", Migration in post-apartheid South Africa: challenges and questions to policymakers, Wa Kabwe-Segatti A. et Landau L., AFD.

World Bank, 2008, Beatting the Odds-Sustaining Inclusion in Mozambique's Growing Economy, Washington : World Bank.

World Bank, 2012, World Development Report 2013. Jobs, Washington : World Bank.

Wuyts M., 1981, Camponeses e economia rural em Moçambique, Maputo : Imprensa Nacional. 